\title{
Una tabla flamenca procedente de Peñaranda de Bracamonte en el Palacio de Bellas Artes de Lille* $^{*}$
}

\author{
Didier MARTENS \\ Université Libre de Bruxelles
}

\begin{abstract}
RESUMEN. El objetivo del presente artículo es atraer la atención sobre una tabla flamenca de principios del siglo XVI que, en 1901-1903, se encontraba todavía en la iglesia de las Carmelitas descalzas de Peñaranda de Bracamonte (Salamanca). En 1946 esta tabla, de la que parecía haberse olvidado la procedencia española, fue adquirida en París por el Museo del Louvre y depositada en el Palais de Beaux-Arts de Lille. Representa un tema bastante raro: Cristo bendiciendo a María. El pintor debió trabajar en Brujas. De hecho, en otra obra que se le puede atribuir, una Virgen con el Niño rodeada de Santa Catalina y Santa Bárbara, conservada en Florencia, se ha inspira en un modelo utilizado con bastante frecuencia en la pintura de Brujas: la Virgo inter virgines atribuida a Hugo Van der Goes.

Palabras clave: Brocado, Lille, Maestro de Hoogstraten, Peñaranda de Bracamonte, Martin Schongauer, Hugo
\end{abstract} van der Goes.

RÉSUMÉ. Le but du présent article est d'attirer l'attention sur un panneau flamand des premières années du XVIème siècle qui, en 1901-1903, se trouvait encore à l'église des Carmelitas Descalzas de Peñaranda de Bracamonte (Salamanque). En 1946, ce panneau, dont la provenance espagnole était, semble-t-il, déjà oubliée, a été acquis à Paris par le Musée du Louvre et mis en dépôt au Palais des Beaux-Arts de Lille. Il représente un thème assez rare: Marie bénissant le Christ. Le peintre a dû travailler à Bruges. En effet, dans une autre oeuvre qui peut lui être attribuée, une Vierge à l'Enfant entourée des saintes Catherine et sainte Barbe, conservée à Florence, il s'est inspiré d'un modèle attesté avec une fréquence remarquable dans la peinture brugeoise: la Virgo inter virgines attribuée à Hugo Van der Goes.

Mots clés: Brocart, Lille, Maître de Hoofstraten, Peñaranda de Bracamonte, Martin Schongauer, Hugo van der Goes.

En una monumental obra, en seis tomos, que acaba de ser publicada, Francis-

\section{-}

*Es un agradable deber agradecer aquí a Florence Gombert, conservadora del Museo de Bellas Artes de Lille, a su asistente Marie-Pierre Rose, a Sonia Caballero Escamilla (Ávila), a Isabelle Lecoq (Bruselas) y a Marta Negro Cobo (Burgos), por la ayuda eficaz que me han prestado en mis investigaciones. Como de costumbre, mi manuscrito se ha beneficiado del senti- do crítico de Jacques de Landsberg, Thierry Lenain y Monique Renault. Deseo igualmente expresar toda mi gratitud al Centro de Estudios de la Pintura del siglo XV en los Países Bajos meridionales y el Principado de Lieja (Bruselas), donde Hélène Mund y Karine Renmans me han acogido muy amablemente. Finalmente, estoy especialmente agradecido a María Dolores Teijeira Pablos (León), por haber asegurado con su competencia la traducción española de mi texto francés. 
co Fernández Pardo ha recordado la avidez internacional de la que no ha dejado de ser víctima el patrimonio artístico español en la época moderna ${ }^{1}$. Desde mediados del siglo XIX, los países precozmente industrializados del Norte de Europa, a los cuales se sumaron pronto los Estados Unidos, aprovecharon su superior peso económico para adquirir obras antiguas en la Península Ibérica. Estos países intentaban llenar con los tesoros del mundo sus recientemente creados museos, como verdadero símbolo de estatus del estado-nación moderno. La rica burguesía local no se quedó atrás. En Londres, Bruselas, París o Berlín buscó reunir, en el espacio privado, las colecciones que pudieran hacer competencia, por su calidad, a las del estado.

España proporcionará a los museos y colecciones extranjeros un gran número de pinturas flamencas de los siglos XV y XVI. Era conocido el gusto de las élites sociales españolas de fines de la Edad Media y principios de los tiempos modernos por el arte de los antiguos Países Bajos. Las obras procedentes de estas regiones constituían objetos de prestigio particularmente apreciados por la corte, la nobleza y los mercaderes. Aprovechando las relaciones comerciales que unían entonces la Península Ibérica con los puertos flamencos, Brujas y Amberes, numerosos castellanos encargaron retablos en el norte o compraron allí obras hechas en serie $^{2}$. Cuando a principios del siglo XIX, bajo la influencia del Romanticismo, se redescubrió en Inglaterra, en Alemania, en

\footnotetext{
-

${ }^{1}$ F. FERNÁNDEZ PARDO, Dispersión y destrucción del Patrimonio artístico español, Madrid, 2007, I-VI.

2 Sobre este tema ver, especialmente, J. LAVALLEYE, Collections d'Espagne, 1-2 (Répertoire des peintures flamandes des XVème et XVIème siècles, 1-2), Amberes, 1953-1958 ; E. BERMEjO MARTíneZ, La pintura de los Primitivos flamencos en España, I-II, Madrid, 1980-1982; F. FERNÁNDEZ PARDO (ed.), Las tablas flamencas en la Ruta jacobea (Catálogo de la exposición), León, Caja España, Edificio Gaudí, 1999.
}

Bélgica y en Francia a los "Primitivos flamencos" y se reconocieron en sus obras las manifestaciones de una fe cristiana simple y naif, todavía "medieval", expresada por temperamentos auténticamente germánicos, anticuarios y mercaderes se entregaron sistemáticamente a la búsqueda de estas obras $^{3}$. Rápidamente se dieron cuenta de que no quedaban más en el norte de Europa. Las destrucciones habían sido numerosas en esta zona en el siglo XVI, como consecuencia de las prácticas iconoclastas de los reformistas. Después, el cambio de gusto, en particular la llegada del clasicismo francés, que hizo despreciar el "Gótico", supuso la desaparición de numerosas pinturas sobre tabla anteriores a 1600 .

En este contexto, España no podía aparecer más que como un "Eldorado" para el comercio de arte internacional. El país, que había escapado completamente al fenómeno iconoclasta y, también en parte, a la dictadura del gusto clásico a la francesa, había conservado en sus iglesias un gran número de trípticos y tablas flamencas de los siglos XV y XVI. Con la complicidad del clero local, los marchantes de arte del norte de Europa buscaron adueñarse de este patrimonio. Sus esfuerzos se vieron a menudo coronados por el éxito, como indica el número impresionante de "primitivos flamencos" en Gran Bretaña, Bélgica, Francia o Alemania de los que es segura la procedencia española.

\footnotetext{
${ }^{3}$ Sobre el redescubrimiento de los "Primitivos flamencos" y sus implicaciones comerciales, ver S. SUlzBERGER, La réhabilitation des Primitifs flamands 1802-1867 (Académie Royale de Belgique, Classe des BeauxArts, Mémoires in -8o, XII, 3), Bruselas, 1961; B. RIDDERBOS, "From Waagen to Friedländer", W. KRUL, "Realism, Renaissance and Nationalism" y T.-H. BORCHERT, "Collecting Early Netherlandish Paintings in Europe and the United States", en Early Netherlandish Paintings. Rediscovery, Reception and Research, Ámsterdam, 2005, pp.218-251, 252-289 y 173-216 respectivamente.
} 
A la nómina de estas obras hay que añadir un panel anónimo conservado en Lille, que hasta ahora apenas había despertado el interés de los historiadores del arte. En las líneas que siguen se intentará establecer su procedencia exacta y determinar la identidad de su autor. El presente artículo pretende de este modo añadir luz a una pieza que, sin ninguna duda, merecería figurar en uno de los volúmenes de Dispersión y destrucción del Patrimonio artístico español....

El Cristo bendiciendo a la Virgen forma parte de la rica colección de "Primitivos flamencos" del Museo de Bellas Artes de Lille $^{4}$ (Lám. 1). Clasificado recientemente por los autores del Répertoire de las Collections $d u$ Nord-Pas-de-Calais como «Países Bajos meridionales, principios del siglo XVI », fue adquirido en 1946 por el Louvre en el Hotel Drouot de París. En ese mismo año fue depositado en Lille. La procedencia de la obra parece haber sido cuidadosamente ocultada por el vendedor. ¿O ya se había olvidado?. Édouard Michel no dice ni una sola palabra sobre el tema en el primer artículo consagrado a la tabla, aparecido en diciembre de $1946^{5}$. No obstante, se puede afirmar que, en los primeros años del siglo $\mathrm{XX}$, el panel estaba todavía encastrado en un retablo de la iglesia de Carmelitas descalzas de Peñaranda de Bracamonte (Sala-

\footnotetext{
${ }^{4}$ Lille, Musée des Beaux Arts; $\mathrm{n}^{\mathrm{o}}$ de inventario P. 1726 (Rf.1946-20). Óleo sobre tabla; 48 x $34 \mathrm{~cm}$. Sobre esta obra, ver últimamente, C. HECK, con la colaboración de H. BOËDEC / D. ADAMS / A. BOllut, Collections $d u$ Nord-Pas-de-Calais. La peinture de Flandre et de France du Nord au XVème et au début du XVIème siècle (Répertoire des peintures flamandes des XVème et XVIème siècles, 5), Bruselas, 2005, n74. La tabla se menciona en A. Brejon De Lavergnee/ J. Foucart/ N. Reynaud, Écoles flamandes et hollandaises (Catalogue sommaire illustré des peintures du Musée du Louvre, I), París, 1979, p.183 («Flandes, principio del siglo XVI»).

${ }^{5}$ E. Michel, «Musées de province : le Christ et la Vierge trônant », Bulletin des Musées de France, 11, 1946, $\mathrm{n}^{\circ} 10, \mathrm{pp} .36-38$.
}

manca). La tabla fue descrita y fotografiada entre 1901 y 1903 por Manuel GómezMoreno, que preparaba entonces la parte del Catálogo Monumental de España consagrada a la provincia de Salamanca. El manuscrito no fue sin embargo publicado hasta $1967^{6}$. La fotografía en blanco y negro reproducida en el libro (Lám. 2) y las dimensiones indicadas por el autor $-47 \times 33$ $\mathrm{cm}$.- permiten identificar sin duda el Cristo bendiciendo a la Virgen de Peñaranda con la tabla de Lille. Gómez Moreno precisa por otra parte, en su descripción, que Cristo "viste túnica morada" y "manto rojo", mientras que la Virgen "viste de blanco y azul". Estos colores se encuentran efectivamente en la tabla de Lille.

Es muy probable que, poco después de ser acabada, la tabla de Lille haya tomado el camino de la Península Ibérica y probablemente incluso de la provincia de Salamanca ${ }^{7}$. Hay que tener en cuenta, sin embargo, que ciertamente no estaba destinada desde un principio a un retablo de la iglesia conventual de las Carmelitas descalzas de Peñaranda de Bracamonte, dado que la fundación del monasterio se remonta al siglo XVII, más concretamente a $1669^{8}$. Exis-

\footnotetext{
${ }^{6}$ M. GÓMEZ-MOREnO MARTíneZ, Catálogo monumental de España. Provincia de Salamanca, Madrid, 1967, pp.453-454, fig.533. Ver también la reedición facsímil (Salamanca, 2003, misma paginación) con introducción de J. R. NiETO GONZÁLEZ. La tabla no se menciona en A. CAsaseca CASAsecA, Catálogo monumental del Partido judicial de Peñaranda de Bracamonte (Salamanca), Madrid, 1984, obra en la que se hace una descripción detallada del patrimonio actual del convento de Carmelitas descalzas (pp.234-270).

7 Los "Primitivos flamencos" conservados en la provincia de Salamanca o que proceden de ella no parecen haber sido hasta el momento objeto de un estudio sistemático. Ver, para un ejemplo recientemente descubierto, A. DiÉGUEZ RoDríGUEZ, "Una Virgen de la leche del taller del Maestro de Frankfurt en el convento salmantino de Dueñas", Boletín del Museo e Instituto "Camón Aznar", 96, 2005, pp.319-329.

${ }^{8}$ Sobre la historia de este monasterio, ver A. CASASECA CASASECA, Op.cit., pp.234-242; C. MÖLleR RE-
} 
te todavía hoy y ha conservado una parte notable de su patrimonio antiguo.

El comentario que Gómez-Moreno hizo de la tabla en el cambio de siglo es bastante representativo del malestar que experimentaban los historiadores del arte ante la pintura de los "Primitivos" del norte de Europa. Al elogiar una "obra de la antigua escuela flamenca, de tal modo selecta y admirable que sin duda salió de manos de alguno de sus grandes maestros", el autor no podía evitar señalar que el rostro de Cristo es "demasiado humano" y que sus pies están "mal dibujado(s)". En cuanto a la Virgen, la encuentra "poco bella". El neoclasicismo condicionaba todavía profundamente la sensibilidad artística de GómezMoreno, que estaba asombrado tanto por la ausencia de idealización en los rostros de Cristo y de María como por el diseño anatómico, más intuitivo que razonado, propio de los "Primitivos flamencos". Esta postura crítica no estaba aislada en el siglo XIX y en la primera mitad del XX. Se leen, en la obra de Eugène Fromentin, reproches similares a propósito de Jan Van Eyck. El autor de Maîtres d'autrefois califica de "fea" la Virgen del canónigo Van der Paele; el Niño sería "un bebé raquítico con poco pelo, copiado al pie de la letra a partir de un pequeño modelo mal alimentado" ${ }^{\prime \prime}$. $Y$ en 1946, si Édouard Michel ya no reprocha a las figuras de la tabla de Lille falta de idealización, no puede evitar destacar en la obra "debilidades, falta de experiencia en el dibujo, curiosas de constatar en ciertos detalles, por ejemplo en la mano con la que Cristo sostiene el globo, en los ropajes a veces torpemente ordenados (...)"10. Es cla-

CONDO / A. M. CARABIAS TORRES, Historia de Peñaranda de Bracamonte (1250-1836), Salamanca - Peñaranda de Bracamonte, 2003, pp.346-364.

${ }^{9}$ E. Fromentin, Les Maîtres d'autrefois. Belgique Hollande (J. Foucart ed.), París, 1965, p.407.

${ }^{10}$ E. MiCHEL, Op.cit., p.36. ro el contraste con la apreciación crítica totalmente positiva formulada en el Répertoire de las Collections du Nord-Pas-de-Calais: los autores hablan sin ambages de una "obra de calidad muy grande, tanto por su nivel de ejecución como por su iconografía"11.

Prudentemente, estos mismos autores no intentaron atribuir la tabla a una personalidad concreta. Édouard Michel había sido más audaz. En su artículo de 1946, afirmaba que el autor de la tabla de Lille había pintado igualmente "una Comida en casa de Simón, pequeña tabla de forma circular que se encontraba en 1933 en el comercio en Amberes, en Sam Artenveld"12. La pintura mencionada es, con toda probabilidad, el tondo flamenco de $19,5 \mathrm{~cm}$. de diámetro que formó parte de la colección De Jaeger en Brujas y que representa efectivamente la Comida de Cristo en la casa de Simón ${ }^{13}$ (Lám. 3). No hay sin embargo ninguna relación estilística manifiesta entre este tondo, que Maurits Smeyers ha atribuido, no sin algunas dudas, a un pintor de Lovaina influido por Dirk Bouts, y la tabla de Lille. ¿Está ésta verdaderamente condenada a convertirse en la única obra de un pintor desconocido?

Yo creo que se puede reconocer la mano del autor de la tabla de Lille en una segunda obra. Conservada en los Uffizi de Florencia, representa a la Virgen con el

${ }^{11}$ C. HECK et al., Op.cit., p.492.

${ }^{12}$ E. Michel, Op.cit., p.37.

${ }^{13}$ Sobre esta obra, ver Catalogue de tableaux des écoles allemande, espagnole, anglaise, flamande, française, hollandaise et italienne du XVIème au XXème siècle (...) (Catálogo de venta), Bruselas, Galería Fiévez, 8 de junio de 1931, no 16; J. DeRAEve, Brugs privé-bezit (Catálogo de la exposición), Brujas, De Croone, 1970, no 5 (con foto en color); M. SMEYERS, nota en Aspekten van de Laatgotiek in Brabant (Catálogo de la exposición), Lovaina, Stedelijk Museum, 1971, pp.214-215, nº LS/9; Catalogue of Important Old Master Paintings (...) (Catálogo de venta), Londres, Sotheby, 11 de julio de 1973, $\mathrm{n}^{\mathrm{o}}$ 21; foto IRPA, Bruselas, M 100855. 
niño, flanqueada a la izquierda por Santa Catalina y a la derecha por Santa Bárbara ${ }^{14}$ (Lám. 4). Sobre el grupo de la Virgen se encuentran dos ángeles volando, que sostienen una corona con las manos.

¿Hay indicios que hablen a favor de la relación entre las dos imágenes?. De entrada destacan afinidades en su composición espacial. El primer plano está ocupado por figuras de talla mediana, dispuestas un poco retranqueadas en relación al marco. El pavimento cuadriculado está delimitado por detrás por un murete que forma un banco. En el eje de simetría vertical de las dos tablas, hacia el cual convergen todas las líneas de fuga de la perspectiva, se encuentra, en el plano medio, un paño de honor. Dibuja un largo rectángulo, relativamente estrecho. Finalmente, tras el muro, se percibe un paisaje muy rico. En general se constata que, en las dos obras, el pintor se las ha ingeniado para subrayar el eje de simetría vertical y multiplicar, en el plano medio, las superficies paralelas de la tabla.

Emparentadas en su concepción espacial, las dos obras lo están igualmente en su vocabulario anatómico. Hay que destacar especialmente las manos largas, de dedos ahusados, de Cristo y de todas las figuras femeninas. Algunas caras parecen repetirse de una pintura a la otra. Se pueden comparar en particular la Virgen con la cabeza inclinada y los ojos medio cerrados de la tabla de Lille con el angelote representado en alto a la izquierda en la tabla florentina (figs. 5 y 6). Tienen básicamente la misma cabeza; la de la criatura celeste parece la reducción de la de María. Destacan en particular los labios carnosos, la frente alta y la nariz saliente con el puente rectilíneo, situado en su prolongación inmediata. Hay que señalar igualmente el detalle de las

\footnotetext{
${ }^{14}$ Florencia, Galleria degli Uffizi; no de inventario 1019; óleo sobre tabla; $84 \times 70 \mathrm{~cm}$.
}

cejas arqueadas, que presentan una cierta disimetría. La ceja izquierda parece, en efecto, tener su origen en el mismo puente de la nariz y "subir" de manera mucho más rápida que la derecha. Estas observaciones valen también para Santa Catalina. Aunque se represente con los grandes ojos abiertos y la cabeza levantada, es difícil no reconocer, en su cara, una versión casi sin modificar de la de la Virgen de la tabla de Lille. Incluso, a pesar de la diferencia de sexo, pueden relacionarse la Santa Bárbara y la figura de Cristo. Sus cabezas proceden de un mismo esquema.

Finalmente, se observan igualmente correspondencias en el vocabulario ornamental. El pavimento representado en primer plano de las tablas de Lille y Florencia presenta de manera destacada cuadrados con una losange colocada sobre su punta y otras subdivididas en cuatro cuadrados. Además, se constata que, en las dos tablas, un mismo motivo de brocado ha servido de modelo al pintor ${ }^{15}$. Tenemos la imagen más completa en el paño de honor de la tabla de Lille (Lám. 7). Un detalle del mismo motivo, ligeramente simplificado y más estrecho, se puede reconocer en la saya de Santa Catalina (Lám. 8). Está delimitado por la abertura de su manto, que se corresponde con el brazo derecho. Destaca el trabajo puntillista de las hojas en las dos tablas.

La tabla de los Uffizi fue atribuida por Max Friedländer al Maestro de Hoogstraten, un pintor que debió formarse en

\footnotetext{
15 Sobre la importancia del brocado en la atribución de pintura de fines de la Edad Media, ver S. H. GODDARD, "Brocade Patterns in the Shop of the Master of Frankfurt: An Accesory to Stylistic Analysis", The Art Bulletin, 67, 1985, pp.401-418. El autor demuestra que en las obras asociadas por la crítica de estilo al Maestro de Frankfurt, se encuentran frecuentemente los mismos motivos de brocado y que no parecen haber sido utilizados por otros talleres. Ver también L. CAMPBELL, National Gallery Catalogues. The Fifteenth Century Netherlandish Schools, Londres, 1998, p.26.
} 
Brujas pero que, además de en esta ciudad, debe haber trabajado también en Amberes $^{16}$. Aunque generalmente aceptada ${ }^{17}$, esta atribución me parece errónea: las caras difieren claramente de las, generalmente más estrechas y de aspecto más anguloso, propias del anónimo de las alas de Hoogstraten $^{18}$. Sin embargo, el cuadro de Florencia parece estar relacionado con Brujas. Su autor se ha inspirado, en efecto, en un modelo documentado de manera casi exclusiva en obras de Brujas de los siglos XV y XVI. Se trata de la famosa Virgo inter virgines en la cual los historiadores del arte ven tradicionalmente una composición inventada por Hugo van der Goes, incluso aunque esta prestigiosa autoría sea fundamentalmente hipotética ${ }^{19}$. De este modelo, co-

${ }^{16}$ M. J. FRIEDLÄNDER, Die altniederländische Malerei VII: Quentin Massys, Berlín, 1929, pp.103, 135, nº 119. Sobre el Maestro de Hoogstraten, ver últimamente $\mathrm{H}$. Mund / N. Goethgebeur, ficha en The Mayer van den Bergh Museum Antwerp (Corpus de la peinture du XVème siècle dans les Pays-Bas méridionaux et la Principauté de Liège, 20), Bruselas, 2003, nº209.

17 Ver especialmente L. COLlOBI RAGGHIANTI, Dipinti fiamminghi in Italia 1420-1570. Catalogo (Musei d'Italia-Meraviglie d'Italia, 24), Bolonia, 1990, no 179.

${ }^{18}$ Ver D. MARTENS, "Transmission et métamorphose d'un modèle: la descendance au XVIème siècle de la Virgo inter virgines attribuée à Hugo van der Goes", Annales de la Société Royale d'Archéologie de Bruxelles, 65, 2002, pp.135-136.

${ }^{19}$ Sobre esta composición, ver F. WINKLER, Das Werk des Hugo van der Goes, Berlín, 1964, pp.155-180; L.CAMPBELL, The Early Flemish Pictures in the Collection of Her Majesty the Queen, Cambridge, 1985, no 32; D.MARTENS, Op.cit., 2002, pp.105-188; S. BUCK, "The Impact of Hugo van der Goes as a Draftsman", Master Drawings, 41, 2003, no 3, pp.229-234; T. KREN, nota en Illuminating the Renaissance. The Triumph of Flemish Manuscript Painting in Europe (Catálogo de la exposición), Los Angeles, The J.Paul Getty Museum, 20032004, no 30; B. DEKEYZER, Herfsttij van de Vlaamse miniatuurkunst: het breviarium Mayer van den Bergh, GanteAmsterdam, 2004, pp.137-143; T. KETELSEN, ficha en Das Gemeimnis des Jan van Eyck. Die frühen niederländischen Zeichnungen und Gemälde in Dresden (Catálogo de la Exposición), Dresde, Residenzschloss, № 19, 2005; B. FRANSEN / P. SYFER-D'OLNE, ficha en Masters with Provisional Names (The Flemish Primitives. Catalogue of Early Netherlandish Painting in the Royal nocido principalmente por las interpretaciones que de él dieron el Maestro de la Leyenda de Santa Lucía y diversos discípulos o imitadores de Gerard David, de Adriaen Isenbrant, de Ambrosius Benson y de los Claeissins, el pintor de la tabla de Florencia ha tomado la figura de Santa Catalina. Con el rostro de tres cuartos inclinado hacia el centro de la imagen, la santa se representa con el brazo derecho doblado, dirigido hacia Cristo, mientras que coge con la mano izquierda una parte de su manto sin mangas. Puede compararse, por ejemplo, con la Santa Catalina que aparece en una versión de la famosa Virgo inter virgines conservada en Roma, en las colecciones de la Academia de San Lucas, donde está atribuida a Adriaen Isenbrant ${ }^{20}$ (Lám. 9). A juzgar por el estilo, se trata ciertamente, en todo caso, de una obra pintada en Brujas. Yo creo que el mismo origen se puede considerar para la tabla florentina y por lo tanto, igualmente, para el Cristo bendiciendo a la Virgen de Lille.

Probablemente no resulta baladí que Édouard Michel sugierese ya vincular esta última obra a la tradición de la miniatura de Gante-Brujas ${ }^{21}$. Sin embargo el contemplaba una atribución a un joven Juan de Flandes, propuesta temeraria que no parece haber suscitado hasta ahora ninguna reacción por parte de los especialistas en este $\operatorname{artista}^{22}$.

A la manera de la Virgo inter virgines de Florencia, el panel de Lille no puede ser

Museums of Fine Arts of Belgium, 4), Bruselas, 2006, p.315. Este último autor pone en duda la existencia de un modelo de Van der Goes común a las diferentes pinturas recogidas por F. WINKLER, L. CAMPBELL y D. MARTENS (ver supra).

${ }^{20}$ L. COLLOBI RAGGHIANTI, op.cit., $\mathrm{n}^{\mathrm{o}} 413$.

${ }^{21}$ E. MiCHEL, op.cit., p.37.

${ }^{22}$ Sobre este artista, ver últimamente C. ISHIKAWA, The "Retablo de Isabel la Católica" by Juan de Flandes and Michel Sittow, Turnhout, 2004; M. P. SILVA MAROTO, Juan de Flandes, Salamanca, 2006. 
considerado enteramente como una creación aparte. Como han descubierto los autores del Répertoire de las Collections $d u$ Nord-Pas-de-Calais, deriva de un grabado de Martin Schongauer que representa igualmente a Cristo bendiciendo a la Virgen ${ }^{23}$ (Lám. 10). “La composición de Lille (...) retoma con exactitud el grupo de la Virgen y Cristo entronizado, y su fidelidad al modelo se muestra hasta en la repetición escrupulosa de cada uno de los pliegues que animan el ropaje de su manto" 24 . Incluso la base plana del trono, prolongada hacia delante por una excrecencia en media luna, se ha inspirado en Schongauer, al igual que, probablemente, la decoración de la parte vertical de la banqueta.

Siempre siguiendo de cerca el modelo grabado, el artista flamenco ha tenido que adaptarlo a lo que debía considerar exigencia propia del medio pictórico. Es por ello que ha reforzado sistemáticamente los efectos ornamentales, introduciendo en su tabla un tejido de brocado, un murete ornado de placas de mármol dispuestas a intervalos regulares, un respaldo de trono con decoración esculpida y un pavimento multicolor. Igualmente ha tenido la precaución de "flamenquizar" su modelo, del que podía difícilmente ignorar su origen extranjero. En este punto, ha suprimido los grandes halos con ribetes y el fondo neutro que, en Schongauer, por la luminosidad que toman de la blancura del papel, recuerdan esas superficies doradas brillantes a las cuales los pintores de más allá del Rin permanecieron mucho más tiempo ligados que

\footnotetext{
${ }^{23}$ Sobre este grabado, ver A. CHÂTELET, ficha en Le beau Martin. Gravures et dessins de Martin Schongauer (Catálogo de la exposición), Colmar, Museé d'Unterlinden, 1991, no G 14; J. NiCOLAISEN, ficha en Martin Schongauer. Druckgraphik im Berliner Kupferstichkabinett (Bilderhefte der Staatlichen Museen Preussischer Kulturbesitz) (Catálogo de la exposición), Berlín, Staatliche Museen, 1991, nº 8 .

${ }^{24}$ C. HECK et al., Op.cit., p.492.
}

sus colegas de los Países Bajos borgoñones ${ }^{25}$. En la tabla de Lille, halos y fondo neutro han cedido el sitio a un paisaje verdeante, animado con pequeños personajes que se dedican a sus ocupaciones, ignorando la presencia divina en primer plano. Un paisaje que, por su carácter de realidad, que parece tomada de lo vivo, se inscribe en la descendencia estética de Jan van Eyck y de su Virgen del Canciller Rolin del Louvre...

La influencia de Schongauer en la pintura flamenca casi no ha suscitado hasta ahora la atención que merece. Postulando una primacía del arte de los antiguos Países Bajos sobre el de Alemania, que no habría finalizado más que con Durero, los investigadores han estado durante mucho tiempo obsesionados por la única cuestión de saber lo que Schongauer debía a los flamencos y no se han preocupado en preguntar de manera sistemática sobre lo que ellos habrían podido tomar de él. Si poseemos, desde 1931, un estudio sobre el impacto que tuvieron los grabados de Alberto Durero en los Países Bajos de la época de Carlos I $^{26}$, nada parecido existe para los de Schongauer. En 1991, con ocasión del coloquio internacional organizado con motivo del 500 aniversario de su muerte, fueron presentadas algunas comunicaciones acerca de la "recepción" del artista alsaciano en Polonia, Hungría e Italia. Ninguna trataba específicamente de "Schongauer en Flandes". En este contexto, hay que saludar, como una loable excepción, la reciente monografía de Stephan Kemperdick, aparecida en 2004, en la cual se dedican varias páginas a las obras

\footnotetext{
${ }^{25}$ Este nexo entre los grandes espacios en blanco de los grabados de Schongauer o Durero y la tradición del fondo dorado ha sido puesto de manifiesto por $\mathrm{W}$. SCHÖNE, Über das Licht in der Malerei, Berlín, 1977, p.105.

26 J. S. HELD, Dürers Wirkung auf die niederländische Kunst seiner Zeit, La Haya, 1931.
} 
flamencas de los siglos XV y XVI que llevan la marca del alsaciano ${ }^{27}$.

Schongauer ha sido el protagonista de un importante proceso de globalización en la cultura figurativa occidental. Desde fines del siglo $\mathrm{XV}$, de Polonia a España, del Norte de Alemania a Italia, se pueden identificar ecos a menudo fieles de sus grabados, los más frecuentes pintados, a veces también esculpidos. Sobre un territorio inmenso que se confundía entonces, poco más o menos, con el de la Europa católica, artistas que se situaban en tradiciones artísticas diferentes, si no antagónicas, utilizaron los mismos modelos. Los antiguos Países Bajos no podían escapar a esta moda más que difícilmente. El tríptico de Melbourne, obra de tres manos de la escuela bruselense pintada entre 1487 y 1492, constituye, en el estado actual del corpus, el primer testimonio bien datado en el arte flamenco de un préstamo indiscutible del alsaciano $^{28}$. Sobre el reverso del ala izquierda figura un Descanso durante la Huida a Egipto ${ }^{29}$. El segundo plano reproduce una

-

${ }^{27}$ S. KEMPERDICK, Martin Schongauer. Eine Monographie, Petersberg, 2004, pp.262-264. Para una aproximación general a la influencia de los grabados alemanes en los pintores de Amberes del primer cuarto del siglo XVI, ver también S. GODDARD, "Assumed Knowledge. The Use of Prints in Early SixteenthCentury Antwerp Workshops", Jaarboek Koninklijk Museum voor Schone Kunsten Antwerpen, 2004/2005, pp.123-139. El autor no dedica, sin embargo, más que una atención muy limitada a Schongauer.

${ }^{28}$ Sobre esta obra, ver C. PERIER-D'IETEREN, “Apports à l'étude du Retable de la Multiplication des Pains de Melbourne, I: le volet des Noces de Cana" y "Apports à l'étude du triptyque de Melbourne, II: la Multiplication des Pains, la Résurrection de Lazare, la Fuite en Égypte et Saint Pierre", en Annales d'Histoire de l'Art et d'Archéologie, 14, 1992, pp-7-25 y 16, 1994, pp.47-78.

${ }^{29}$ Las dos caras de esta ala han sido atribuidas de manera convincente por A. CARLIER al Maestro de los Retratos principescos. Ver "Oeuvres de jeunesse du Maître de la Légende de sainte Madeleine: nouvelles attributions", en Le Maître au Feuillage brodé. Démarches d'artistes et méthodes d'attribution d'euvres à un peintre parte del grabado de Schongauer que ilustra el mismo tema, especialmente los cinco ángeles que curvan una palma de manera que José pueda coger los dátiles ${ }^{30}$.

A fines del siglo $\mathrm{XV}$ y principios del XVI, la enorme difusión de los grabados de Schongauer le confería una autoridad particular, resultado de esta omnipresencia. Debían ser considerados modelos iconográficos fiables, tanto por los pintores como por su clientela: cuando hacía falta representar un tema poco común, se podía apoyar sin temor en la interpretación que de él había dado el alsaciano. El tema de Cristo bendiciendo a la Virgen es, de modo general, mucho más raro que el de la Coronación de la Virgen, con el cual está emparentado. Deseoso de evitar todo error iconográfico y quizá incluso por demanda expresa del patrón, el anónimo de Brujas que pintó la tabla de Lille calcó prudentemente su Cristo bendiciendo a la Virgen sobre el grabado por el alsaciano. Sin embargo lo "flamenquizó" hasta tal punto que ha sido necesario esperar hasta el año 2005 para que el préstamo haya sido reconocido.

anonyme des anciens Pays-Bas du XVème siècle. Colloque organisé par le Palais des Beaux-Arts de Lille les 23 et 24 juin 2005, Deauville, 2007, pp.87-98.

${ }^{30}$ Sobre este grabado, ver A. CHÂTELET, nota en $L e$ beau Martin. Gravures et dessins, Op.cit., nº G 8; J. NICOLAISEN, ficha en Martin Schongauer. Druckgraphik, Op.cit., $\mathrm{n}^{\mathrm{o}} 3$ c. 


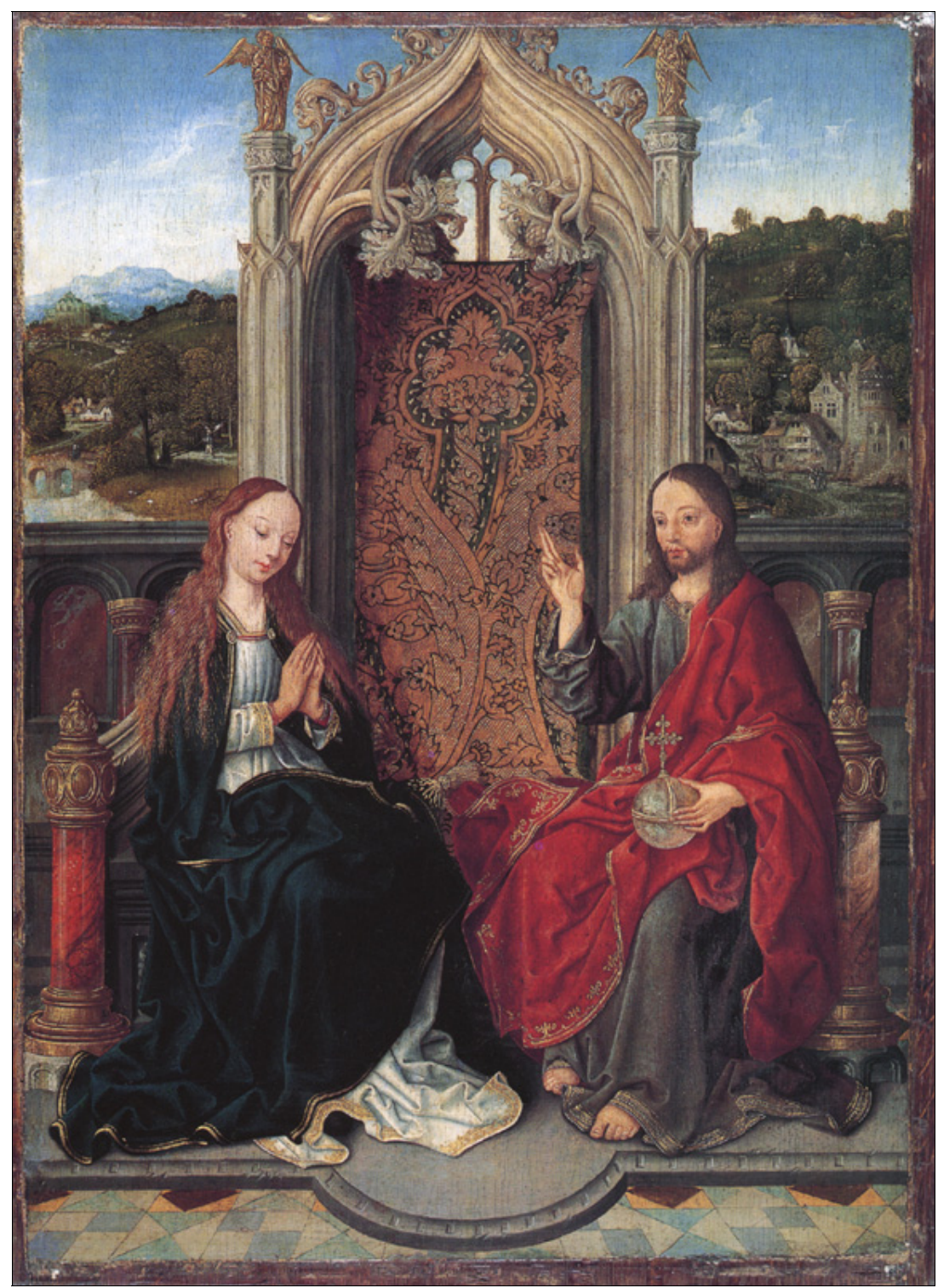

- Lám. 1. Maestro de Brujas, principios del siglo XVI: Cristo bendiciendo a la Virgen; Lille, Musée des Beaux-Arts (cliché RMN, París). 


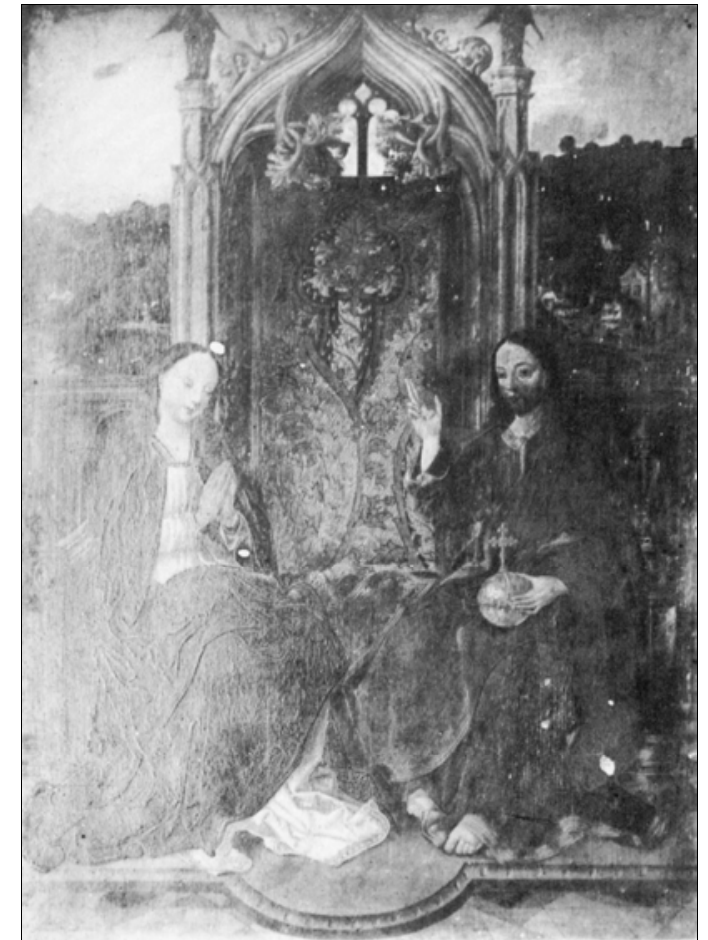

- Lám. 2. Maestro de Brujas, principios del siglo XVI: Cristo bendiciendo a la Virgen; Lille, Musée des Beaux-Arts (antigua fotografía tomada de M. GómeZ-Moreno MARTíneZ, Catálogo Monumental de España. Provincia de Salamanca, Madrid, 1967, fig.533).

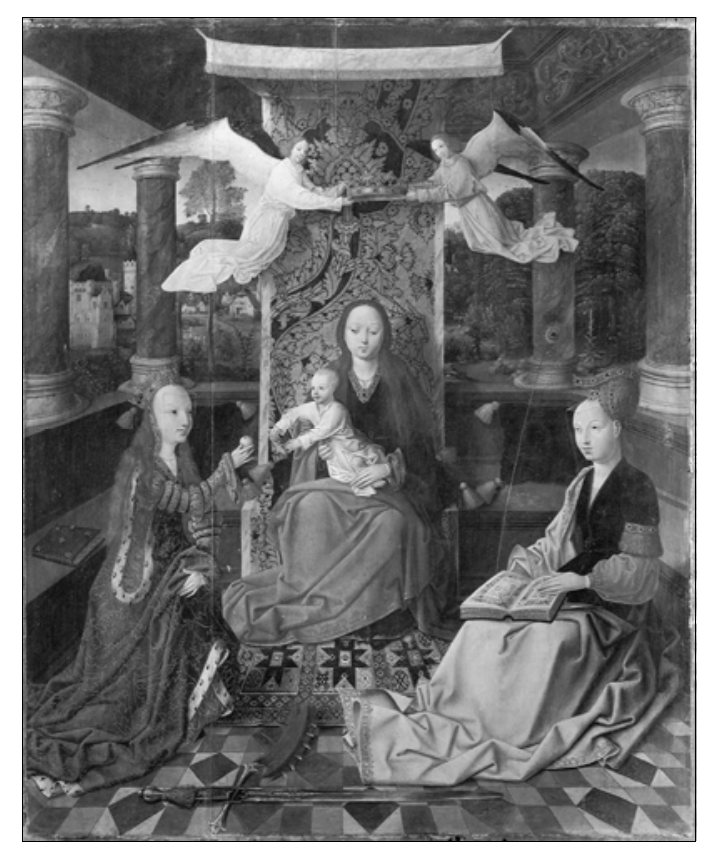

- Lám. 4. Maestro de Brujas, principios del siglo XVI: Virgo inter virgines; Florencia, Galleria degli Uffizi (cliché IRPA, Bruselas).

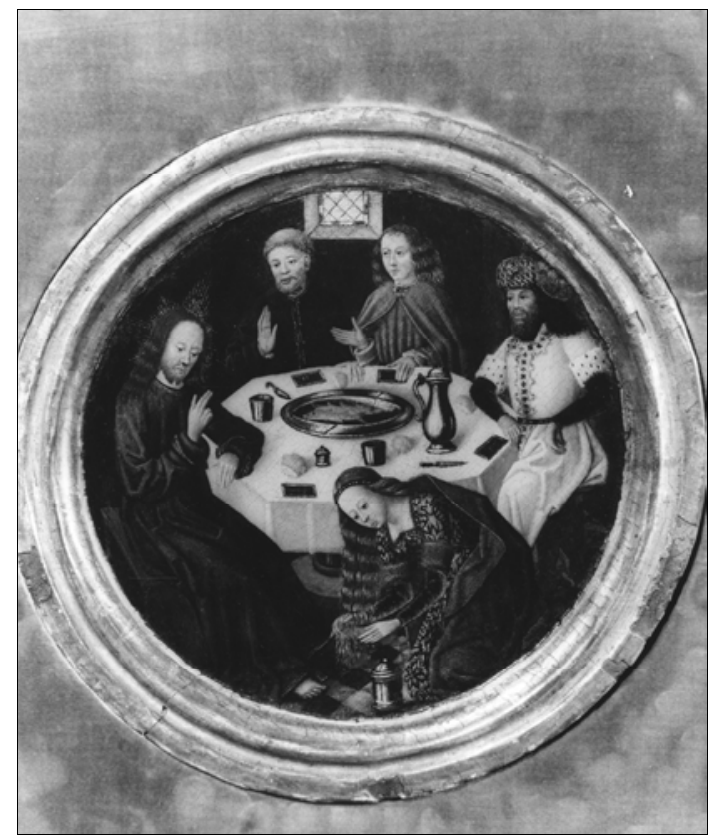

- Lám. 3. Maestro Brabanzón (?), fines del siglo XV: Comida de Cristo en casa de Simón; antiguamente en Brujas, colección A. de Jaeger (cliché IRPA, Bruselas).

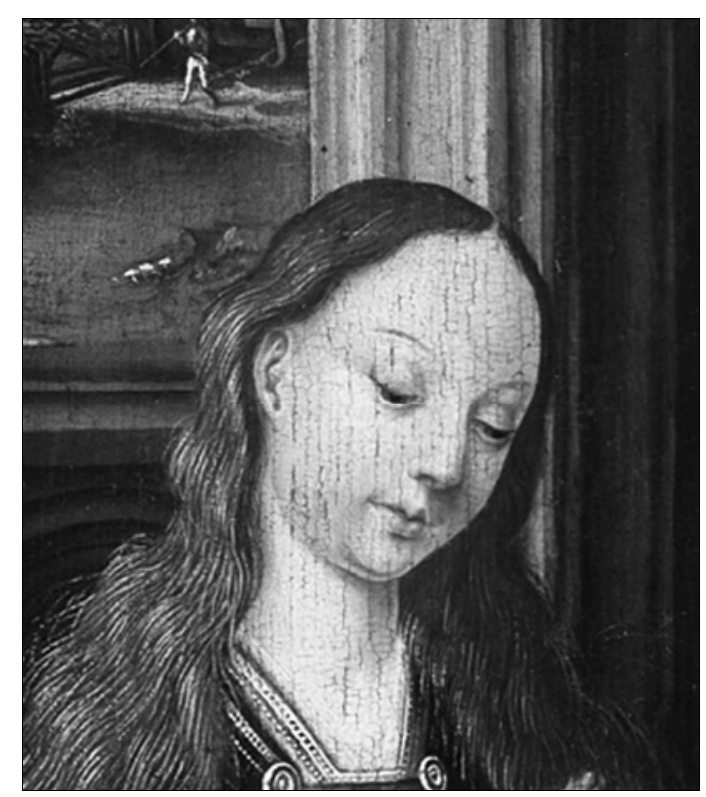

- Lám. 5. Detalle de la figura 1 (cliché IRPA, Bruselas). 


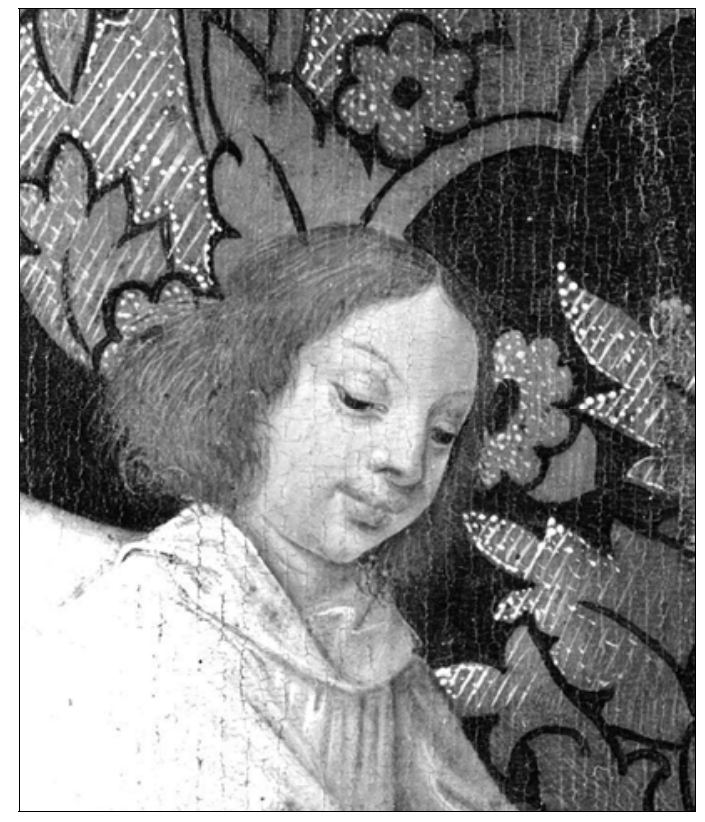

- Lám. 6. Detalle de la figura 4 (cliché IRPA, Bruselas).

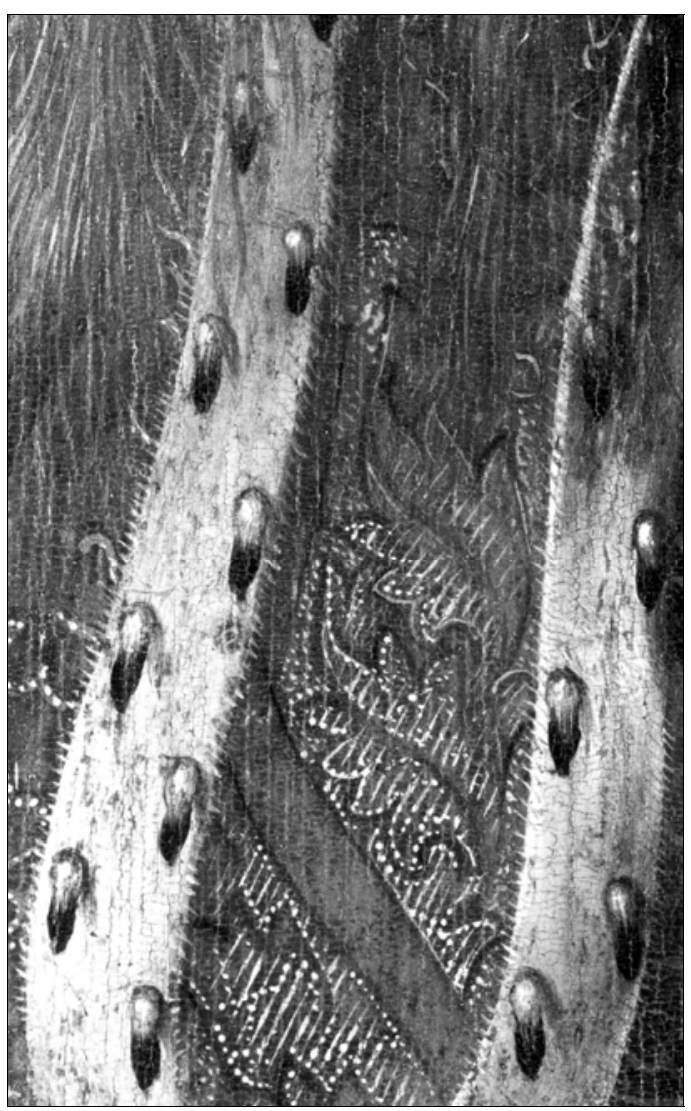

- Lám. 8. Detalle de la figura 4 (cliché IRPA, Bruselas)

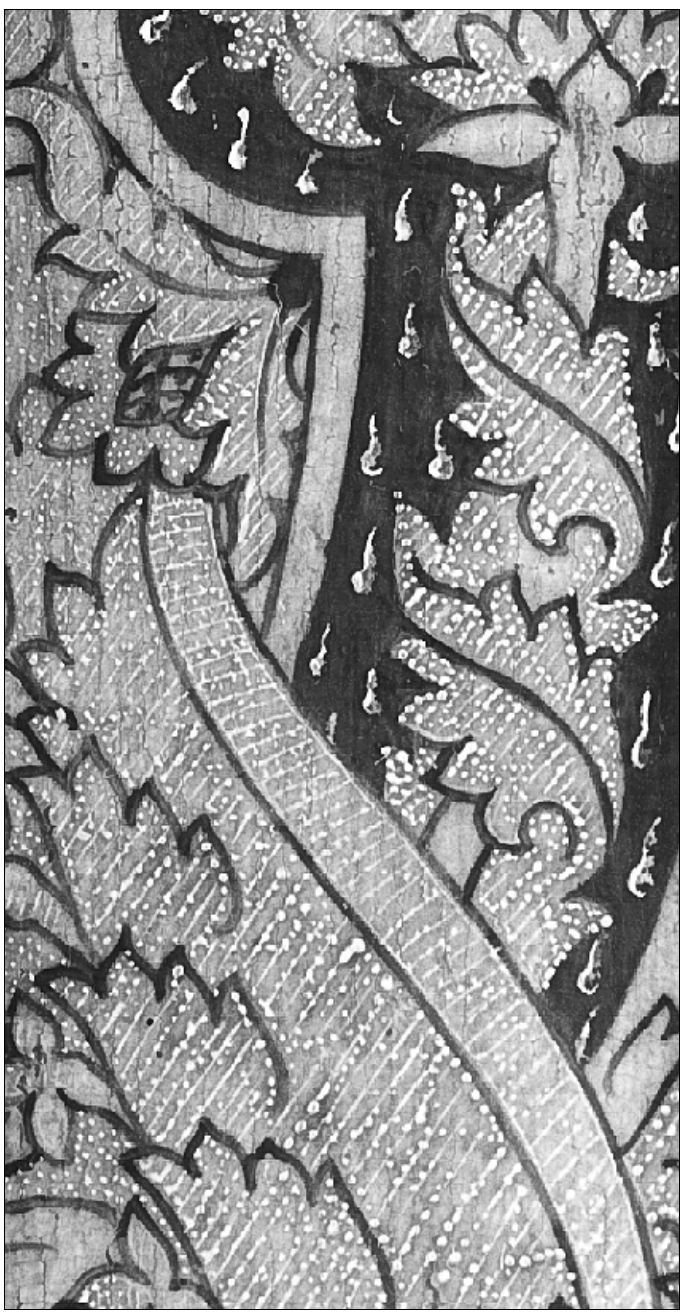

- Lám. 7. Detalle de la figura 1 (cliché IRPA, Bruselas). 


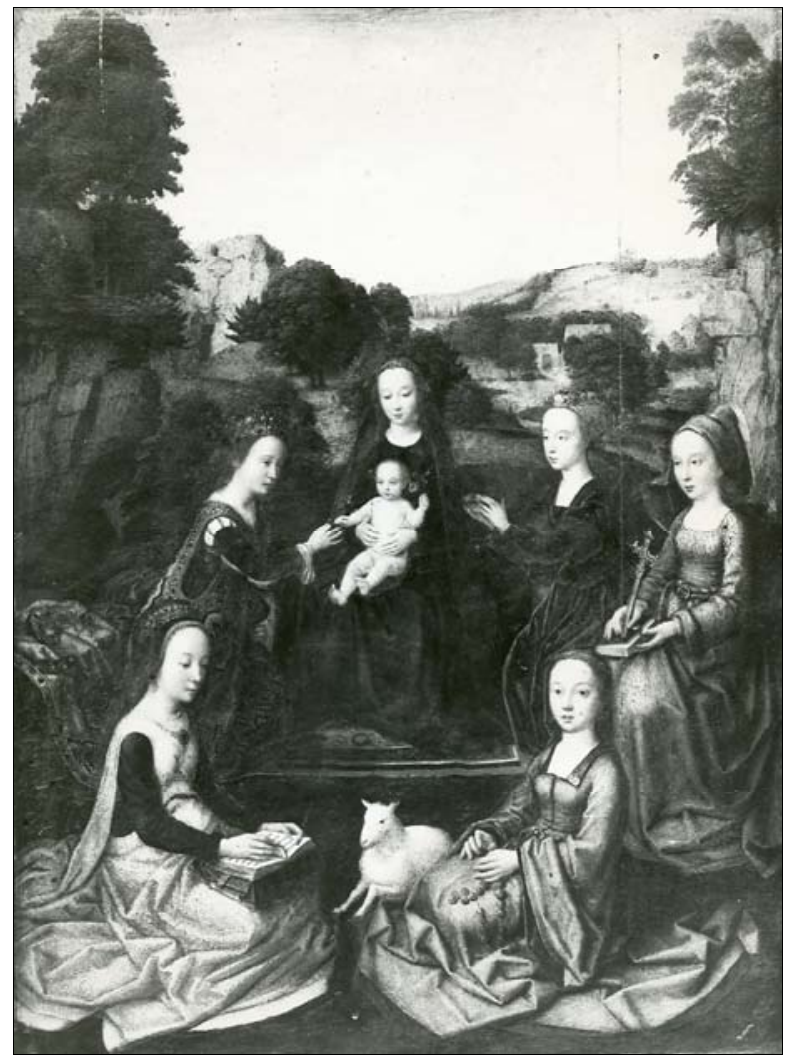

- Lám. 9. Adriaen Isenbrant (círculo de): Virgo inter virgines; Roma, Accademia de San Luca (cliché Gabinetto fotografico nazionale, Roma).

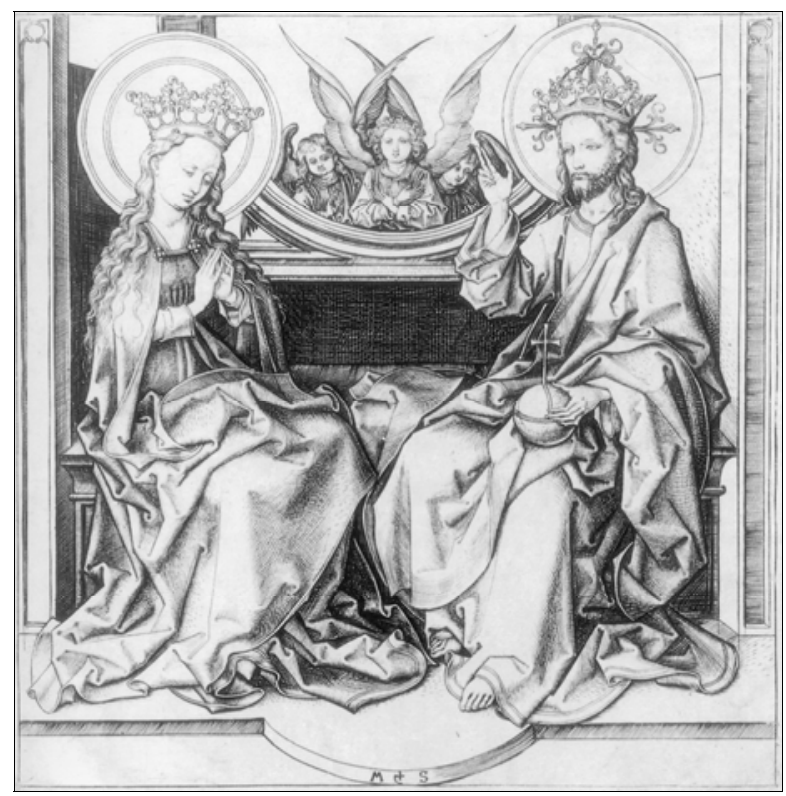

- Lám. 10. Martin Schongauer: Cristo bendiciendo a la Virgen; Dresde. Staatliche Kunstsammlungen, Kupferstichkabinett. 